\title{
Consumo de tabaco en cinco ciudades de Colombia, Encuesta Mundial de Tabaquismo en Jóvenes, 2007
}

\author{
Constanza Pardo ${ }^{1}$, Marion Piñeros ${ }^{2}$ \\ 1 Grupo de Vigilancia Epidemiológica del Cáncer, Instituto Nacional de Cancerología, Bogotá, D.C., Colombia \\ 2 Grupo del Área Salud Pública, Instituto Nacional de Cancerología, Bogotá, D.C., Colombia
}

Introducción. El consumo de tabaco es un problema de salud pública en el mundo. En Colombia se le atribuyen más de 17.000 muertes al año. Es necesario vigilar este factor de riesgo prevenible para las enfermedades crónicas y el cáncer.

Objetivo. Estimar la prevalencia de tabaquismo y de otros factores que influyen en el uso de tabaco, en cinco ciudades de Colombia.

Materiales y métodos. La encuesta fue implementaba por el Instituto Nacional de Cancerología en 2007, en colegios públicos y privados; fue anónima, voluntaria y autodiligenciada por estudiantes de 13 a 15 años, seleccionados por muestreo bietápico por conglomerados. El análisis estadístico se realizó con el software SUDAAN. Se consideraron diferencias estadísticamente significativas si p era menor de 0,05).

Resultados. La edad promedio de inicio en el consumo de tabaco en las cinco ciudades fue de 11,9 años. La prevalencia del consumo de cigarrillo estuvo entre $7,4 \%$ y $34,1 \%$; el riesgo de inicio entre los no fumadores estuvo entre $12,3 \%$ y $32 \%$. Entre $40 \%$ y $60 \%$ de los estudiantes estuvieron expuestos a humo ambiental de tabaco en lugares públicos y alrededor de $70 \%$ estuvieron expuestos a publicidad de tabaco. Entre $40 \%$ y $69 \%$ quieren dejar de fumar. Aproximadamente, $80 \%$ consigue cigarrillos en una tienda o supermercado, sin interesar la edad. Entre 34\% y 54\% recibieron información en los colegios sobre los peligros de fumar.

Conclusiones. Las altas prevalencias del consumo en cuatro de las cinco ciudades sugieren intervenciones dirigidas principalmente a los componentes de prevención, espacios libres de humo y control de la publicidad.

Palabras clave: tabaco, adolescente, factores de riesgo, vigilancia epidemiológica, Colombia.

\section{Teenage tobacco consumption in five Colombian cities}

Introduction. The consumption of tobacco is a public health problem around the world. In Colombia, each year approximately 17,000 deaths are attributed to smoking. The monitoring of tobacco consumption is necessary to estimate population risk for chronic diseases and cancer.

Objective. The prevalence of smoking was estimated and factors assessed that influence the use of tobacco among youths.

Materials and methods. The survey was implemented by the Colombian National Cancer Institute in 2007, in public and private schools located in 5 cities in Colombia. The survey was anonymous, voluntary and self-processed by students 13 to 15 years of age and the participants selected by a multi-stage sampling process. Statistical analysis was performed with SUDAAN software. Differences in proportions were considered statistically significant at the $p<0.05$ level.

Results. The average age of onset for tobacco consumption in the five cities was 11.9 years. The prevalence of cigarette smoking varied between $7.4 \%$ and $34.1 \%$ among the cities and susceptibility to initiate smoking among non smokers was between $12.3 \%$ to $32.0 \%$. Between $40 \%$ and $60 \%$ of students were exposed to secondhand smoke in public places. Approximately $70 \%$ were exposed to indirect tobacco advertising. Between $40 \%$ and $69 \%$ of students who currently smoke cigarettes reported that they wanted to stop smoking. Approximately $80 \%$ of students who currently smoke cigarettes were not refused when they purchased cigarettes in a store or supermarket. Finally, $34 \%$ to $54 \%$ of students reported having been taught in school about the harmful effects of smoking.

Conclusions. The high prevalence of consumption in four of the five cities suggested interventions that aim primarily at prevention components, smoke-free spaces and advertising control.

Key words: Tobacco, adolescent, risk factors, epidemiologic surveillance, Colombia. 
El consumo de tabaco es un problema de salud pública en el mundo y constituye un factor de riesgo para seis de las ocho principales causas de mortalidad en el mundo, como son: la enfermedad pulmonar obstructiva crónica, el cáncer de tráquea, bronquios y pulmón, la cardiopatía isquémica, las enfermedades cerebro-vasculares, las infecciones de las vías respiratorias inferiores y la tuberculosis (1). En 2015 se esperan cerca de 6,4 millones de muertes atribuibles al tabaco con un aumento considerable, principalmente, en países de ingresos bajos y medios (2). En Colombia, se estima que más de 17.000 muertes al año son atribuibles al consumo de tabaco (3). Se han reportado diversos factores que influyen en el uso de tabaco, entre ellos están la exposición al humo de tabaco ajeno, la exposición indirecta a la publicidad de tabaco y la facilidad de acceso por bajos precios.

Teniendo en cuenta que el tabaco constituye el factor de riesgo prevenible y modificable más importante para las enfermedades crónicas y el cáncer, que los adolescentes y los jóvenes son grupos de población más vulnerables de adquirir conductas de riesgo y que en estas edades la modificación de hábitos es más fácil, es necesario para el país vigilar y supervisar su uso y los factores relacionados de manera periódica y sistemática para establecer las actividades de control del tabaco.

En Colombia, la prevalencia de tabaquismo en adolescentes se ha establecido por medio de diferentes encuestas con representatividad nacional y departamental (4-7). En dos de las encuestas cuyos resultados son comparables, la prevalencia de uso experimental incrementó de 12,7\% en 1993 (4) a 18\% en 1998 (5). Además, en 2001 se aplicó la primera Encuesta Mundial de Tabaquismo en Jóvenes en colegios públicos de Bogotá para vigilar el uso de tabaco en los jóvenes escolares, la cual mostró una de las prevalencias más altas para la región $(29,8 \%)(8)$.

Esta situación llevó a que Colombia ratificara el convenio marco para el control del tabaco de la Organización Mundial de la Salud $(9,10)$, el cual

\footnotetext{
Correspondencia:

Constanza Pardo, Grupo de Vigilancia Epidemiológica del Cáncer, Instituto Nacional de Cancerología, Calle 1 № 9-85, Bogotá, D.C., Colombia.

Teléfono: 334 1111, extensión 4111; fax: 3341353

cpardo@cancer.gov.co y tachapardo@yahoo.com

Recibido: 09/09/09; aceptado:29/06/10
}

insta al país a legislar y a tomar medidas relativas a la reducción de la demanda y de la oferta para proteger la salud de la población $(11,12)$. Con el fin de ayudar a los países a cumplir los compromisos de dicho convenio, la Organización Mundial de la Salud diseñó un paquete de medidas, entre las cuales el supervisar y vigilar el consumo de tabaco constituyen una actividad transversal (1).

La aplicación de la Encuesta Mundial de Tabaquismo en Jóvenes permite cumplir con esta actividad, además de fortalecer el sistema de vigilancia epidemiológica de factores de riesgo en jóvenes escolares, para orientar las intervenciones del control de tabaco en el país y contribuir con información para el Sistema Mundial de Vigilancia de Tabaco y poder hacer comparaciones con el mundo (13). Esta encuesta hace parte del Sistema Mundial de Vigilancia de Tabaco, sistema iniciado por la Organización Mundial de la Salud, los Centers for Disease Control and Prevention, y la Canadian Public Health Association, y ha sido promovida para la vigilancia del control de tabaco y de los programas en los distintos países (14).

En Colombia es la segunda vez que se desarrolla la encuesta, con una mayor cobertura, cuyo objetivo fue estimar la prevalencia de tabaquismo en cinco ciudades de Colombia (Bogotá, Bucaramanga, Cali, Manizales y Valledupar), teniendo en cuenta que son grandes ciudades que representan las distintas macrorregiones del país y en donde existen registros de cáncer con base en la población.

En este artículo se presentan los resultados de las estimaciones de la prevalencia de consumo de tabaco y otros factores, como exposición al humo ambiental de tabaco, riesgo de fumar, exposición a publicidad pro-tabaco, cesación, acceso y disponibilidad de información sobre tabaco obtenida en los colegios, realizada en 2007 en los estudiantes de 13 a 15 años de las cinco ciudades.

\section{Materiales y métodos}

La Encuesta Mundial de Tabaquismo en Jóvenes fue implementada por el Instituto Nacional de Cancerología, con la colaboración técnica de la Organización Mundial de la Salud, la Organización Panamericana de la Salud y los Centers for Disease Control and Prevention. La encuesta se aplicó en los colegios públicos y privados de cinco ciudades de Colombia (Bogotá, Bucaramanga, Cali, Manizales y Valledupar), en los grados 7 a 10, e incluyó estudiantes de 13 a 15 años. 


\section{Muestra}

El cálculo de la muestra y las estimaciones se realizaron con base en la metodología estandarizada por los Centers for Disease Control and Prevention (13). La selección de las ciudades se hizo por conveniencia para correlacionar la información de la encuesta con la información de los registros de cáncer con base en la población existentes en estas ciudades.

El marco muestral fue de 2.228 colegios públicos $(36 \%)$ y privados $(64 \%)$ existentes a 2006 , para un total de 663.703 estudiantes en los grados 7 a 10, con una distribución mayor en los públicos (62\%) que en los privados (38\%).

La muestra probabilística e independiente para cada ciudad fue bietápica por conglomerados. En la primera etapa se seleccionaron los colegios con una probabilidad proporcional al número de estudiantes matriculados en los grados 7 a 10. En la segunda etapa se seleccionaron las clases mediante un muestreo aleatorio simple, en que todos los estudiantes pertenecientes a estas clases eran elegibles para el estudio. La muestra incluyó 191 colegios entre públicos $(66 \%)$ y privados (34\%). Para las ciudades de Bogotá y Cali, se seleccionaron 50 colegios, para Valledupar, 31, y para Bucaramanga y Manizales, 30 , respectivamente.

\section{Recolección de información}

El proyecto, los cuestionarios y el formato de notificación a los padres fueron aprobados por el Comité de Ética del Instituto Nacional de Cancerología de Colombia.

Las actividades de campo se iniciaron con las muestras y la selección de los colegios y las clases. Inicialmente se estableció contacto con las Secretarías de Educación y de Salud en cada una de las ciudades, para obtener respaldo territorial y se definió un cronograma de implementación para cada una de las ciudades.

Los colegios seleccionados se visitaron con el fin de presentar la metodología de la encuesta y obtener la autorización para la aplicación de la misma. Por otra parte, los padres de familia fueron notificados antes de aplicar la encuesta; sin embargo, cada estudiante participó de forma voluntaria en el momento de responderla.

La recolección de la información se hizo en cada una de las clases de los colegios seleccionados mediante un cuestionario anónimo, con el apoyo de recurso humano entrenado para la aplicación de la encuesta.

El cuestionario diseñado y validado por los Centers for Disease Control and Prevention fue adaptado para Colombia, con la realización de una traducción pareada y una prueba piloto con la versión en español, para verificar la comprensión de las preguntas. Se definieron 76 preguntas, de las que 49 eran comunes a todos los países; los módulos seleccionados fueron: consumo de cigarrillo y otros productos de tabaco, conocimientos y actitudes, exposición al humo ambiental de tabaco, cesación, exposición a medios de comunicación y publicidad, actividades contra el consumo de tabaco en los colegios e información de tipo demográfico.

Se tomó como definición de "fumador actual" al estudiante que fumó, por lo menos, un día en los últimos 30 días. Posteriormente, los Centers for Disease Control and Prevention realizaron el procesamiento de datos (escaneo, limpieza, edición y ponderación).

\section{Análisis de datos}

El análisis de los datos incluyó el cálculo de tres índices de respuesta (escolar, estudiantil y general), la ponderación de los datos por la cual se pueden expandir los resultados de la muestra hacia toda la población de estudiantes, por ciudad y en el rango de edad, y la estimación de los intervalos de confianza de $95 \%$.

La tasa de respuesta escolar se calculó con el número de colegios que aceptaron participar sobre el número de colegios muestreados. La tasa de respuesta estudiantil se calculó con el número de estudiantes que aceptaron participar sobre el total de estudiantes seleccionados. La respuesta total se calculó como el producto de la tasa de respuesta escolar y la respuesta estudiantil.

Se utilizó un factor de ponderación que se calculó como el inverso de la probabilidad de selección para cada etapa y se ajustó por falta de respuesta para cada colegio, clase y estudiante. La generación de los factores de ponderación y el cálculo de los estimadores junto con sus intervalos de confianza, se hicieron conforme con la estrategia muestral descrita, con el programa SUDAAN (15).

\section{Resultados}

En total, 6.437 estudiantes entre los 13 y 15 años completaron la encuesta, con predominio del sexo femenino. En las cinco ciudades la tasa de respuesta escolar estuvo por encima de $92 \%$ y la 
tasa de respuesta estudiantil estuvo en un rango de $85,9 \%$ a $91,3 \%$, y la más baja fue en la ciudad de Cali. Manizales fue la ciudad con mejor tasa de respuesta general (cuadro 1).

\section{Consumo de tabaco}

La edad promedio de inicio en el consumo de tabaco para las cinco ciudades fue de 11,9 años. La mayor prevalencia de haber fumado alguna vez en la vida se encontró en Manizales (68,8\%) y la menor en Valledupar (26,7\%), con diferencias significativas en general y por sexo para las ciudades de Bucaramanga y Cali. La prevalencia de fumador actual de cigarrillo osciló entre $34,1 \%$ y $7,4 \%$, con la tasa más alta en Manizales y la más baja en Valledupar; sin embargo, Cali presentó una proporción significativamente mayor en hombres $(31,4 \%)$ que en mujeres $(22,2 \%)$.

Manizales, Bogotá y Valledupar fueron las ciudades con la mayor proporción de estudiantes que empezaron a fumar antes de los 10 años. Entre los que nunca habían fumado y que tenían el riesgo de inicio en el tabaquismo, Bogotá tuvo la tasa más alta (32\%), seguida de Manizales (30\%), mientras que en Valledupar solamente el $12,3 \%$ piensa fumar en el año siguiente. En las cinco ciudades el consumo de cigarrillo fue significativamente mayor que el uso de otros productos de tabaco, aunque Manizales mostró la prevalencia más alta de estudiantes que actualmente usan algún producto del tabaco distinto del cigarrillo (7,5\%) (cuadro 2).

\section{Exposición a humo ambiental de tabaco}

Los estudiantes más expuestos al humo ambiental de tabaco en su casa fueron los de Manizales (40,4\%), ciudad con una prevalencia significativamente mayor que Bucaramanga, Cali y Valledupar; sin embargo, en esta última ciudad la exposición en casa fue significativamente mayor en mujeres $(25,4 \%)$ que en hombres (16,8\%).

El mayor porcentaje de estudiantes con padres fumadores estuvo en Manizales (61,8\%) y el menor en Valledupar (21,6\%). La exposición en lugares públicos varió entre $61,6 \%$ y $40,1 \%$. Manizales fue la ciudad donde los estudiantes reportaron tener un mayor número de amigos que fuman $(25,1 \%)$, seguida de Cali $(21,9 \%)$ y Bogotá $(21,3 \%)$. Más de $75 \%$ de los estudiantes en las cinco ciudades piensan que debe prohibirse fumar en todos los lugares públicos y más de $80 \%$ de los no fumadores, proporción significativamente mayor a la de fumadores que apoyan esta medida (cuadro 3).

\section{Exposición indirecta a publicidad de tabaco}

En las cinco ciudades alrededor de $70 \%$ de los estudiantes dijeron haber visto algún mensaje contra el consumo de tabaco en los medios de comunicación y haber estado expuestos a publicidad de tabaco en vallas y revistas o periódicos en el último mes. Los estudiantes de Valledupar estuvieron significativamente menos expuestos a este tipo de publicidad en vallas $(65,1 \%)$ y revistas 0 periódicos $(61,7 \%)$, con respecto a las otras cuatro ciudades. En general, el porcentaje de estudiantes que reportaron tener algún objeto con el logotipo de una marca de cigarrillos, estuvo entre 8,2\% (Valledupar) y 14,2\% (Manizales). En relación con la oferta de cigarrillos gratis por alguna compañía tabacalera, Bucaramanga tuvo una prevalencia significativamente más alta $(32,1 \%)$ que Bogotá $(19,2 \%)$ y Valledupar $(22,2 \%)$ (cuadro 4$)$.

Cuadro 1.Tasas de respuesta en estudiantes de 13 a 15 años, Encuesta Mundial de Tabaquismo en Jóvenes, Colombia, 2007.

\begin{tabular}{|c|c|c|c|c|c|}
\hline & \multicolumn{5}{|c|}{ Ciudad } \\
\hline & Bogotá & Bucaramanga & Cali & Manizales & Valledupar \\
\hline \multicolumn{6}{|c|}{ Tasa de respuesta (\%) } \\
\hline Colegios & 93,8 & 93,3 & 91,8 & 93,3 & 93,5 \\
\hline Estudiantes $_{2}$ & 89,5 & 85,9 & 88,4 & 91,3 & 86,6 \\
\hline Totalq & 83,9 & 80,2 & 81,2 & 85,2 & 81,0 \\
\hline \multicolumn{6}{|l|}{ Grupo de edad } \\
\hline 13 a 15 años & 1.235 & 1.455 & 1.268 & 1.326 & 1.153 \\
\hline \multicolumn{6}{|l|}{ Sexo } \\
\hline Hombres (\%) & 44,2 & 41,6 & 43,7 & 45,7 & 45,0 \\
\hline Mujeres (\%) & 55,8 & 58,4 & 56,3 & 54,3 & 55,0 \\
\hline
\end{tabular}

${ }_{1}$ La tasa de respuesta escolar se calculó como: número de colegios participantes / número de colegios seleccionados * 100 ${ }_{2}$ La tasa de respuesta estudiantil se calculó como: número de estudiantes participantes/ número de estudiantes seleccionados * 100 La tasa de respuesta total se calculó como: tasa de respuesta escolar * tasa de respuesta estudiantil. 
Cuadro 2. Prevalencia de consumo de tabaco y de otros productos según sexo, en estudiantes de 13 a 15 años, Encuesta Mundial de Tabaquismo en Jóvenes, Colombia, 2007.

Consumo de cigarrillo y otros productos del tabaco

\begin{tabular}{|c|c|c|c|c|c|c|}
\hline Ciudad & (\%, IC 95\%) & $\begin{array}{l}\text { Alguna vez han } \\
\text { fumado cigarrillos }\end{array}$ & $\begin{array}{l}\text { Fumador actual } \\
\text { de cigarillos }\end{array}$ & $\begin{array}{c}\text { Fumador } \\
\text { actual de otros } \\
\text { productos del tabaco }\end{array}$ & $\begin{array}{l}\text { Entre fumadores, } \\
\text { los que probaron } \\
\text { por primera vez } \\
\text { cigarrillo antes } \\
\text { de los diez años }\end{array}$ & $\begin{array}{l}\text { No fumadores } \\
\text { que quieren } \\
\text { empezar a fumar } \\
\text { el próximo año }\end{array}$ \\
\hline \multirow[t]{3}{*}{ Bogotá, D.C. } & Total & $57,1(52,3-61,7)$ & $26,2(22,5-30,3)$ & $5,0 \quad(3,7-6,8)$ & $13,5(10,4-17,4)$ & $32,0 \quad(28,7-35,5)$ \\
\hline & Hombres & $58,7(54,1-63,1)$ & $25,4 \quad(21,0-30,3)$ & $6,7 \quad(4,7-9,7)$ & $17,2(11,0-25,8)$ & $31,9(25,9-38,6)$ \\
\hline & Mujeres & $55,6(48,1-62,8)$ & $26,6(20,9-33,1)$ & $3,6(2,4-5,4)$ & $10,5(7,0-15,3)$ & $31,8(27,5-36,5)$ \\
\hline \multirow[t]{3}{*}{ Bucaramanga } & Total & $43,3(37,1-49,8)$ & $15,5(11,7-20,1)$ & $3,2(2,2-4,6)$ & $12,1 \quad(9,6-15,3)$ & $22,3(19,0-26,0)$ \\
\hline & Hombres & $51,6(45,5-57,7)$ & $19,3(14,4-25,3)$ & $4,2(2,3-7,5)$ & $10,4(7,9-13,5)$ & $20,1 \quad(15,2-26,1)$ \\
\hline & Mujeres & $37,0 \quad(31,0-43,4)$ & $12,1 \quad(8,3-17,3)$ & $2,2(1,3-3,9)$ & $13,8(9,9-18,9)$ & $23,0(18,8-27,9)$ \\
\hline \multirow[t]{3}{*}{ Cali } & Total & $55,0 \quad(51,3-58,6)$ & $26,2(23,9-28,7)$ & $6,6(5,0-8,6)$ & $11,4(8,7-14,8)$ & $27,4(22,9-32,3)$ \\
\hline & Hombres & $63,4 \quad(58,2-68,3)$ & $31,4 \quad(26,9-36,2)$ & $9,0(6,5-12,3)$ & $12,2(8,9-16,6)$ & $27,3(20,9-34,8)$ \\
\hline & Mujeres & $48,6 \quad(44,2-52,9)$ & $22,2(18,5-26,3)$ & $4,6(3,2-6,6)$ & $10,8(6,8-16,7)$ & $27,7(21,8-34,5)$ \\
\hline \multirow[t]{3}{*}{ Manizales } & Total & $68,8(64,8-72,6)$ & $34,1 \quad(30,3-38,1)$ & $7,5(5,6-10,2)$ & $14,4(11,7-17,6)$ & $30,0(24,0-36,8)$ \\
\hline & Hombres & $70,6(65,4-75,3)$ & $33,0 \quad(28,7-37,7)$ & $8,2 \quad(4,9-13,3)$ & $16,2(11,5-22,2)$ & $24,9(16,1-36,4)$ \\
\hline & Mujeres & $67,3(63,1-71,2)$ & $34,8(29,2-40,8)$ & $6,5(4,4-9,5)$ & $12,6(10,0-15,7)$ & $34,4(26,7-43,1)$ \\
\hline \multirow[t]{3}{*}{ Valledupar } & Total & $26,7 \quad(23,0-30,7)$ & $7,4(5,2-10,6)$ & $3,0(2,0-4,4)$ & $13,2(10,1-17,1)$ & $12,3(9,6-15,5)$ \\
\hline & Hombres & $29,1 \quad(24,1-34,6)$ & $6,0(4,0-8,8)$ & $2,4(1,2-4,4)$ & $11,5(7,8-16,7)$ & $10,3(7,1-14,7)$ \\
\hline & Mujeres & $24,5(20,2-29,5)$ & $8,5(4,9-14,3)$ & $3,5(2,1-5,6)$ & $14,6(9,8-21,1)$ & $13,2(10,3-16,9)$ \\
\hline
\end{tabular}

IC: intervalo de confianza

Cuadro 3. Prevalencia de exposición a humo ambiental de tabaco según sexo, en estudiantes de 13 a 15 años, Encuesta Mundial de Tabaquismo en Jóvenes, Colombia, 2007.

\begin{tabular}{|c|c|c|c|c|c|c|}
\hline \multirow[b]{2}{*}{ Ciudad } & \multicolumn{6}{|c|}{ Exposición a humo ambiental de tabaco } \\
\hline & (\%, IC 95\%) & $\begin{array}{c}\text { Uno o los } \\
\text { dos padres fuman }\end{array}$ & $\begin{array}{c}\text { Todos o la mayoría } \\
\text { de los amigos } \\
\text { fuman }\end{array}$ & $\begin{array}{c}\text { Exposición al humo } \\
\text { de otros en } \\
\text { el hogar }\end{array}$ & $\begin{array}{l}\text { Exposición } \\
\text { al humo } \\
\text { de otros en } \\
\text { lugares públicos }\end{array}$ & $\begin{array}{c}\text { Piensan que } \\
\text { debe prohibirse } \\
\text { fumar en } \\
\text { lugares públicos }\end{array}$ \\
\hline \multirow[t]{3}{*}{ Bogotá, D.C. } & Total & $50,5(46,6-54,4)$ & $21,3(17,7-25,4)$ & $26,2(23,4-29,3)$ & $56,1 \quad(52,7-59,4)$ & $77,7 \quad(73,7-81,3)$ \\
\hline & Hombres & $46,3(41,3-51,3)$ & $17,8(12,9-24,1)$ & $25,3(21,1-30,0)$ & $55,1 \quad(50,0-60,1)$ & $79,5(73,7-84,3)$ \\
\hline & Mujeres & $54,2(48,8-59,4)$ & $24,1 \quad(20,4-28,2)$ & $27,0(23,9-30,4)$ & $56,9(52,6-61,1)$ & $76,2(71,5-80,2)$ \\
\hline \multirow[t]{3}{*}{ Bucaramanga } & Total & $35,8(32,1-39,8)$ & $16,4(12,4-21,3)$ & $24,0(22,1-26,1)$ & $52,6(49,8-55,3)$ & $84,6(81,1-87,5)$ \\
\hline & Hombres & $38,4(34,5-42,5$ & $18,1 \quad(13,1-24,5)$ & $23,9(20,3-28,0)$ & $53,5(49,9-57,0)$ & $83,1 \quad(79,0-86,5)$ \\
\hline & Mujeres & $33,8(28,7-39,4)$ & $15,1 \quad(11,1-20,2)$ & $23,7 \quad(20,2-27,6)$ & $52,0 \quad(48,0-56,0)$ & $85,7 \quad(81,8-88,8)$ \\
\hline \multirow[t]{3}{*}{ Cali } & Total & $41,9(38,6-45,4)$ & $21,9(18,1-26,2)$ & $24,2(21,5-27,2)$ & $52,7(49,8-55,5)$ & $77,8(74,5-80,7)$ \\
\hline & Hombres & $46,2(40,0-52,5)$ & $18,3(15,5-21,4)$ & $22,3(18,2-27,0)$ & $52,6(47,2-58,0)$ & $76,6 \quad(72,4-80,2)$ \\
\hline & Mujeres & $39,1 \quad(35,3-43,0)$ & $24,8(19,4-30,9)$ & $25,7 \quad(21,9-29,8)$ & $52,7 \quad(49,7-55,7)$ & $79,0 \quad(74,7-82,7)$ \\
\hline \multirow[t]{3}{*}{ Manizales } & Total & $61,8(57,7-65,9)$ & $25,1 \quad(21,4-29,3)$ & $40,4 \quad(37,4-43,5)$ & $61,6(58,1-65,0)$ & $75,3(72,0-78,3)$ \\
\hline & Hombres & $62,3 \quad(56,7-67,5)$ & $22,9(18,7-27,7)$ & $38,3(33,0-43,8)$ & $59,7 \quad(54,2-65,0)$ & $76,1 \quad(71,7-79,9)$ \\
\hline & Mujeres & $60,5(56,1-64,9)$ & $27,0 \quad(21,7-33,0)$ & $41,5(38,6-44,5)$ & $63,3(60,3-66,1)$ & $74,3(69,4-78,5)$ \\
\hline \multirow[t]{3}{*}{ Valledupar } & Total & $21,6(18,6-25,0)$ & $4,1(2,4-6,7)$ & $21,7(18,9-24,9)$ & $40,1 \quad(37,9-42,3)$ & $90,3(88,5-91,9)$ \\
\hline & Hombres & $17,9(14,1-22,4)$ & $3,8(1,9-7,4)$ & $16,8 \quad(13,7-20,5)$ & $37,2(33,8-40,7)$ & $89,3 \quad(85,5-92,2)$ \\
\hline & Mujeres & $24,1 \quad(19,9-29,0)$ & $4,3(2,7-6,8)$ & $25,4 \quad(21,4-29,9)$ & $41,9(39,0-44,9)$ & $91,1 \quad(88,7-93,0)$ \\
\hline
\end{tabular}

IC: intervalo de confianza

\section{Cesación}

El porcentaje de estudiantes que quieren dejar de fumar fue mayor en Bucaramanga $(69,4 \%)$ y Cali $(68,8 \%)$ y menor en Valledupar $(42,9 \%)$; sin embargo, el $60 \%$ de los estudiantes en todas las ciudades reportaron haber recibido ayuda alguna vez para dejar de fumar. En Cali hubo diferencias significativas por sexo, con un mayor porcentaje de hombres $(80,2 \%)$ que quieren dejar de fumar. La prevalencia de los que necesitan fumar a primera hora de la mañana, entre los fumadores actuales, en general fue baja para las cinco ciudades; la mayor prevalencia la informó Valledupar, únicamente en mujeres $(5,1 \%)$, seguida de Manizales $(4,1 \%)$ (cuadro 5).

\section{Acceso}

Por lo menos, el $60 \%$ de los estudiantes de Bucaramanga, Cali y Manizales compraron cigarrillos en una tienda o supermercado, cifras significativamente mayores que en Valledupar. En Bogotá, cerca de $40 \%$ de los fumadores tuvieron 
Cuadro 4. Prevalencia de exposición indirecta a publicidad de tabaco según sexo, en estudiantes de 13 a 15 años, Encuesta Mundial de Tabaquismo en Jóvenes, Colombia, 2007.

\begin{tabular}{|c|c|c|c|c|c|c|}
\hline \multicolumn{7}{|c|}{ Medios de comunicación } \\
\hline Ciudad & $\%$, IC 95\%) & $\begin{array}{l}\text { Durante el mes } \\
\text { pasado vio algún } \\
\text { mensaje contra } \\
\text { el tabaco } \\
\text { en los medios }\end{array}$ & $\begin{array}{c}\text { Durante el mes } \\
\text { pasado vio algún } \\
\text { anuncio de cigarrillos } \\
\text { en vallas }\end{array}$ & $\begin{array}{c}\text { Durante el mes } \\
\text { pasado vio algún } \\
\text { anuncio o promoción } \\
\text { de cigarrillos en } \\
\text { periódicos o revistas }\end{array}$ & $\begin{array}{c}\text { Tenían algún } \\
\text { objeto con el } \\
\text { logo de cigarrillos }\end{array}$ & $\begin{array}{c}\text { Alguna vez le } \\
\text { ofrecieron cigarrillos } \\
\text { gratis por una } \\
\text { compañía tabacalera }\end{array}$ \\
\hline \multirow[t]{3}{*}{ Bogotá, D.C. } & Total & $69,1 \quad(66,2-71,8)$ & $75,8 \quad(72,5-78,8)$ & $75,2(71,0-79,0)$ & $11,0(8,8-13,7)$ & $19,2(16,5-22,1)$ \\
\hline & Hombres & $68,6(64,8-72,1)$ & $74,86(69,5-79,4)$ & $76,3 \quad(69,7-81,8)$ & $13,5(10,4-17,4)$ & $20,1 \quad(16,5-24,3)$ \\
\hline & Mujeres & $69,9(65,9-73,6)$ & $76,6(73,2-79,6)$ & $74,3(70,0-78,2)$ & $9,0 \quad(6,3-12,6)$ & $18,3(15,1-22,0)$ \\
\hline \multirow[t]{3}{*}{ Bucaramanga } & Total & $66,6(63,1-69,8)$ & $74,1 \quad(70,6-77,3)$ & $75,2(72,2-78,0)$ & $11,7 \quad(9,4-14,6)$ & $32,1 \quad(28,8-35,5)$ \\
\hline & Hombres & $67,1 \quad(61,6-72,1)$ & $74,3(69,3-78,7)$ & $78,2(74,1-81,9)$ & $14,0(11,0-17,7)$ & $35,2(31,5-39,0)$ \\
\hline & Mujeres & $66,2(62,1-70,1)$ & $734,6(69,7-77,3)$ & $73,2(69,1-76,9)$ & $9,5(7,1-12,6)$ & $29,4(25,0-34,3)$ \\
\hline \multirow[t]{3}{*}{ Cali } & Total & $69,9(65,7-73,8)$ & $73,4 \quad(71,0-75,6)$ & $73,5(69,9-76,8)$ & $11,2(9,2-13,7)$ & $26,8(23,0-31,0)$ \\
\hline & Hombres & $71,7(66,5-76,3)$ & $74,4 \quad(69,8-78,5)$ & $72,0 \quad(66,1-77,3)$ & $13,4(10,5-17,0)$ & $27,6(23,6-31,9)$ \\
\hline & Mujeres & $68,1 \quad(62,8-72,9)$ & $72,6(69,8-75,3)$ & $74,3(70,5-77,8)$ & $9,3(7,4-11,7)$ & $26,2(21,4-31,7)$ \\
\hline \multirow[t]{3}{*}{ Manizales } & Total & $72,9(70,6-75,2)$ & $75,3(71,8-78,5)$ & $72,4(68,6-75,9)$ & $14,2(12,0-16,8)$ & $29,0 \quad(26,3-31,8)$ \\
\hline & Hombres & $72,1 \quad(67,6-76,2)$ & $74,2(69,2-78,6)$ & $73,6(68,5-78,2)$ & $16,5(13,3-20,4)$ & $26,1 \quad(22,7-29,9)$ \\
\hline & Mujeres & $73,2(69,3-76,8)$ & $77,0 \quad(72,9-80,6)$ & $71,7(66,9-76,1)$ & $12,3(9,7-15,4)$ & $31,6(28,0-35,5)$ \\
\hline \multirow[t]{3}{*}{ Valledupar } & Total & $66,1 \quad(61,6-70,3)$ & $65,1 \quad(62,1-68,0)$ & $61,7(58,7-64,6)$ & $8,2(6,9-9,8)$ & $22,2(18,9-25,8)$ \\
\hline & Hombres & $64,2(59,5-68,7)$ & $62,8(58,3-67,0)$ & $61,7(57,1-66,1)$ & $9,9(7,7-12,7)$ & $21,7 \quad(17,4-26,6)$ \\
\hline & Mujeres & $67,8(61,9-73,2)$ & $67,0(63,0-70,7)$ & $61,5(56,5-66,3)$ & $6,6(4,7-9,2)$ & $22,3(19,0-25,9)$ \\
\hline
\end{tabular}

IC: intervalo de confianza

Cuadro 5. Prevalencia de cesación, acceso a la compra de productos de tabaco en tiendas y actividades contra el tabaco en colegios según sexo, en estudiantes de 13 a 15 años, Encuesta Mundial de Tabaquismo en Jóvenes, Colombia, 2007.

\begin{tabular}{|c|c|c|c|c|c|c|c|}
\hline \multicolumn{8}{|c|}{ Cesación, acceso y actividades contra el tabaco en colegios } \\
\hline Ciudad & $(\%$, IC $95 \%)$ & $\begin{array}{c}\text { Fumadores } \\
\text { actuales } \\
\text { que desean dejar } \\
\text { dejar de fumar }\end{array}$ & $\begin{array}{l}\text { Fumadores } \\
\text { actuales que han } \\
\text { recibido ayuda para } \\
\text { dejar de fumar }\end{array}$ & $\begin{array}{c}\text { Fumadores } \\
\text { actuales que } \\
\text { necesitan fumar } \\
\text { a primera hora } \\
\text { de la mañana }\end{array}$ & $\begin{array}{c}\text { Fumadores } \\
\text { actuales que } \\
\text { normalmente } \\
\text { compran cigarrillos } \\
\text { en una tienda }\end{array}$ & $\begin{array}{c}\text { Fumadores } \\
\text { actuales que } \\
\text { compran cigarrillos } \\
\text { en una tienda y no } \\
\text { se les niega por } \\
\text { causa de su edad }\end{array}$ & $\begin{array}{l}\text { Durante este } \\
\text { año escolar, les } \\
\text { enseñaron en } \\
\text { clase sobre los } \\
\text { los peligros } \\
\text { de fumar }\end{array}$ \\
\hline \multirow[t]{3}{*}{ Bogotá, D.C. } & Total & $64,7(56,5-72,1)$ & $60,5(53,9-66,8)$ & 0 & $51,7(42,8-60,6)$ & $59,5(48,8-69,3)$ & $34,6(27,8-42,1)$ \\
\hline & Hombres & $65,5(54,2-75,3)$ & $64,3(52,4-74,6)$ & 0 & $54,1(42,0-65,8)$ & $57,7(45,6-68,9)$ & $34,6(27,2-42,8)$ \\
\hline & Mujeres & $63,1(49,9-74,6)$ & $56,9(50,1-63,4)$ & 0 & $49,2(38,5-60,0)$ & $60,4(42,9-75,6)$ & $34,7(27,1-43,2)$ \\
\hline \multirow[t]{3}{*}{ Bucaramanga } & a Total & $69,4(57,9-78,8)$ & $63,4(54,9-71,2)$ & $1,6(0,5-5,1)$ & $61,8(52,9-70,0)$ & $82,2(69,9-90,2)$ & $47,1 \quad(43,3-51,0)$ \\
\hline & Hombres & $67,4(51,9-79,8)$ & $64,0(53,1-73,7)$ & $1,9(0,5-7,5)$ & $65,7(53,4-76,2)$ & $79,2(66,1-88,2)$ & $46,0 \quad(39,7-52,5)$ \\
\hline & Mujeres & $71,7(55,8-83,6)$ & $61,0(53,0-68,5)$ & $1,3(0,1-10,3)$ & $59,9(46,6-71,9)$ & $84,8 \quad(66,6-94,0)$ & $47,6(43,9-51,4)$ \\
\hline \multirow[t]{3}{*}{ Cali } & Total & $68,8(61,6-75,2)$ & $62,3(55,6-68,6)$ & $1,7(0,5-5,8)$ & $62,9(55,8-69,5)$ & $80,0(70,5-86,9)$ & $35,1 \quad(29,1-41,7)$ \\
\hline & Hombres & $80,2(69,3-87,9)$ & $69,2(62,9-74,9)$ & 0 & $64,2(54,3-73,1)$ & $71,5(59,2-81,3)$ & $39,6(32,3-47,4)$ \\
\hline & Mujeres & $58,7(50,4-66,4)$ & $56,4(46,3-66,0)$ & $2,8(0,7-10,8)$ & $61,6(53,1-69,6)$ & $89,0(77,5-95,0)$ & $31,6(25,7-38,2)$ \\
\hline \multirow[t]{3}{*}{ Manizales } & Total & $67,8(57,2-76,9)$ & $60,8(53,2-67,8)$ & $4,1(1,9-8,6)$ & $64,3(59,4-69,0)$ & $82,0(75,6-87,0)$ & $53,9(43,9-63,6)$ \\
\hline & Hombres & $71,5(58,6-81,6)$ & $64,5(56,5-71,8)$ & $4,1 \quad(1,9-8,6)$ & $67,8(60,7-74,2)$ & $80,7(67,9-89,2)$ & $59,5(50,7-67,7)$ \\
\hline & Mujeres & $64,4(53,9-73,8)$ & $57,9(47,8-67,3)$ & $4,3(1,4-12,8)$ & $61,4 \quad(53,2-69,0)$ & $84,0(77,0-89,2)$ & $49,2(37,0-61,6)$ \\
\hline \multirow[t]{3}{*}{ Valledupar } & Total & $42,9(17,6-72,6)$ & $60,8(43,2-76,0)$ & $5,1 \quad(0,6-30,9)$ & $45,9(36,6-55,5)$ & $97,0(78,3-99,7)$ & $43,9(37,9-50,1)$ \\
\hline & Hombres & $73,7(43,6-91,0)$ & $64,0(44,7-79,7)$ & 0 & $62,0(45,3-76,3)$ & $93,1 \quad(60,2-99,2)$ & $40,8(34,0-48,0)$ \\
\hline & Mujeres & $30,3(8,1-68,2)$ & $58,0 \quad(34,2-78,5)$ & $7,9(0,9-44,3)$ & $36,1 \quad(20,9-54,8)$ & 100 & $46,5(39,7-53,4)$ \\
\hline
\end{tabular}

algún tipo de restricción al acceso con una diferencia significativa en relación con las otras cuatro ciudades (cuadro 5).

\section{Actividades contra el consumo de tabaco en colegios}

En general, menos de la mitad de los estudiantes dijeron haber recibido información en clase durante el año escolar sobre los peligros de fumar, con un rango entre $34,6 \%$ (Bogotá) y 53,9\% (Manizales) (cuadro 5).

\section{Discusión}

En general, la aplicación de la presente Encuesta Mundial de Tabaquismo en Jóvenes mostró en todas las ciudades unas altas tasas de respuesta escolar y estudiantil, comparables con las de otros países de la región de las Américas, como los obtenidos en México y Estados Unidos (16), a pesar de la solicitud del consentimiento a los padres que podría haber limitado la respuesta. La aplicación de una metodología de estimación por muestra probabilística, el uso de un cuestionario validado 
y la realización de actividades estandarizadas para la implementación de la encuesta, generaron estimaciones de calidad y altos índices de respuesta para cada una de las ciudades, lo que confirma la validez del estudio.

Los resultados mostraron una prevalencia alta de consumo de tabaco. En conjunto para las cinco ciudades, la prevalencia de vida promedio de consumo de cigarrillos o algún producto de tabaco fue de $55,5 \%$ y la prevalencia promedio de fumador actual de cigarrillos (21,9\%) fue mayor que las prevalencias observadas entre los adolescentes $(2,5 \%)$ y los adultos $(12,8 \%)$ con la Encuesta Nacional de Salud de 2007 y otras encuestas realizadas en Colombia (4-7); y fue menor que la obtenida con la encuesta de tabaquismo realizada en $2001(8,16)$. A pesar de no tener metodologías comparables en algunas encuestas, las diferencias encontradas son muy altas, lo que podría atribuirse justamente a la metodología empleada. Algunos estudios han confirmado la fiabilidad y validez del autorreporte en encuestas que indagan sobre tabaquismo en adolescentes (17-19), como fue el caso de la presente encuesta.

En comparación con otros países de América Latina, la prevalencia promedio de fumador actual es similar a la de Argentina y menor que la de México en 2006 (27,1\%), Chile en 2003 (33,9\%) y menor que la prevalencia total para la región de las Américas $(13,16)$. Se ha descrito que distintos factores, como son la alta exposición a humo ambiental en el entorno familiar y social, la exposición a publicidad y promoción de tabaco y la facilidad en el acceso, influyen o condicionan el inicio de tabaquismo en los escolares, lo cual puede explicar el comportamiento de la alta prevalencia de fumadores en Colombia (20-22).

La prevalencia promedio de probabilidad de inicio en el consumo de cigarrillo $(23,1 \%)$ fue más alta que la de fumador actual y muy cercana a la prevalencia para la región de las Américas (23,9\%) (16), lo que hace pensar en la alta vulnerabilidad de los estudiantes a las presiones de los amigos y a la influencia que puede ejercer la publicidad (23). Sumado a esto, la vulnerabilidad fue mayor entre las mujeres $(24,2 \%)$ que entre en los hombres $(21,2 \%)$, situación que es similar para el mundo, donde el uso de tabaco está siendo mas común entre mujeres, principalmente en los países de bajos ingresos (24), además de ser blanco actual de las compañías tabacaleras (25).

Es importante considerar que uno de cada 10 estudiantes experimentó con cigarrillo antes de los 10 años y es probable que esta situación no mejore, mientras no se regule la venta de productos de tabaco a menores de edad y se piense que las campañas antitabaco también se deberían dirigir a los estudiantes que se encuentran en los dos últimos años de la educación primaria. Esta exposición puede reducirse con el cumplimiento de las normas sustantivas para la protección integral de los niños y los adolescentes de Colombia (26).

Otros hallazgos relevantes del presente estudio se relacionan con la percepción de los estudiantes sobre la exposición al humo ambiental de tabaco en la casa o en lugares públicos (53\%), la cual está cercana a la estimada para la región de las Américas (55\%) (13), y la prohibición de fumar en lugares públicos como restaurantes, autobuses, escuelas, discotecas, gimnasios y centros deportivos, entre otros. En relación con lo anterior, se ha demostrado que los espacios libres de humo reducen el consumo de tabaco en 3\% a $4 \%$ (27) y que la reducción de la exposición a humo de segunda mano debe ser un elemento esencial de todo programa para el control de tabaco (28). Estos resultados sirven de base para que las secretarías de salud del país implementen diversas estrategias como lo define el Plan Nacional de Salud Pública en su objetivo de disminuir las enfermedades no transmisibles y las discapacidades, y vigilar el cumplimiento de la reglamentación nacional en relación con la prohibición del consumo de tabaco en lugares públicos $(9,29)$, y ofrecer educación sobre los peligros del humo ambiental de tabaco y las ventajas que traen los espacios libres de humo como estrategia para disminuir la aceptabilidad social (30).

Los países que ratificaron el convenio marco para el control de tabaco están obligados a legislar en relación con la restricción de la publicidad y tener un mayor número de advertencias sanitarias en las cajetillas de cigarrillos (Artículo 13), además de limitar el acceso a la compra en tiendas (Artículo 16) $(14,28)$.

Los resultados del presente estudio en términos de la elevada exposición a la publicidad y el acceso de los menores de edad a los productos de tabaco en tiendas y supermercados sin restricción alguna, indican la necesidad de legislar sobre estos dos artículos en el corto plazo, para lo cual recientemente el país sancionó la Ley 1335 , la cual definió disposiciones sobre la venta de productos de tabaco y prevención del consumo en menores de edad, relativas a la publicidad y empaquetado, 
prohibición de promoción y patrocinio, y garantizar los derechos de las personas no fumadoras (14).

Esto pone de manifiesto la necesidad de continuar con estas preguntas en futuras aplicaciones de la Encuesta Mundial de Tabaquismo en Jóvenes en Colombia, con el fin de evaluar la adopción de estas decisiones normativas. Al respecto, llama la atención cómo en Estados Unidos solamente $8 \%$ de los escolares compran los cigarrillos en una tienda (16). Por otra parte, se ha evidenciado que en los niños, la exposición a la promoción del tabaco antes de usarlo, además de la falta de barreras en cuanto acceso y venta, aumenta el riesgo de un inicio temprano $(20,22)$. A pesar de esta situación, se encuentra amplia conciencia en los estudiantes sobre el riesgo que corren con el consumo de tabaco, debido a que, por lo menos, $60 \%$ de los estudiantes fumadores quieren dejar de hacerlo. Se ha evidenciado que la búsqueda de ayuda y asesoría mejora las tasas de abandono; sin embargo, en sólo nueve países hay plenamente disponibles servicios para tratar la dependencia de tabaco (1).

El porcentaje de estudiantes que refirieron recibir enseñanza en los colegios sobre los peligros del tabaco fue bajo en todas las ciudades, lo que es similar a los resultados obtenidos en la Encuesta Mundial de Tabaquismo en Jóvenes de otros países $(16,31)$. Aunque algunos estudios resaltan el rol que tienen los colegios en la identificación de comportamientos no favorables como el consumo de tabaco, así como en la promoción de la salud $(21,32)$, la información disponible hace referencia principalmente a las intervenciones en el espacio escolar, más que a recibir información en clases. Los resultados de estas intervenciones son contradictorios y hastael momento es difícil demostrar o excluir un efecto benéfico de las intervenciones basadas en el suministro de información (33).

En consonancia con el Artículo 20 del convenio marco para el control del tabaco que regula sobre la vigilancia en tabaco, la aplicación de la encuestas a jóvenes estudiantes de forma periódica y sistemática es un primer paso para evaluar el comportamiento de la magnitud, los patrones, los factores determinantes y las consecuencias del consumo y exposición a humo de tabaco en cinco de las principales ciudades del país.

Se identificaron dos limitaciones en este estudio, que vale la pena aclarar. En primer lugar, la encuesta es representativa únicamente para estudiantes entre los 13 y 15 años para cada una de las ciudades encuestadas y, por lo tanto. no pueden extrapolarse los resultados a los adolescentes del país. Por otra parte, la encuesta fue autoadministrada, por lo que pudo haber un sesgo de información por algunos factores como el sexo, la edad y la interpretación de las preguntas en la encuesta (34).

La mayor cobertura de la Encuesta de Tabaquismo en Jóvenes realizada en 2007 ofrece una línea de base para evaluar distintas actividades para el control de tabaco en Colombia. Las altas tasas de participación escolar y estudiantil, y el uso de la metodología definida por los Centers for Disease Control and Prevention de Atlanta, utilizada por muchos países del mundo, hacen que los resultados de esta encuesta sean representativos para los estudiantes de 7 a 10 grado de los colegios públicos y privados de las ciudades encuestadas.

Los resultados mostraron altas prevalencias de consumo de tabaco en cuatro de las cinco ciudades encuestadas y gran riesgo de los estudiantes al inicio en el hábito de fumar en el próximo año. Un hallazgo importante fue la alta exposición de los escolares al humo ambiental de tabaco en lugares públicos y la baja restricción para la compra de cigarrillos en los menores de edad. Esto confirma la necesidad de establecer o fortalecer iniciativas de salud pública en este grupo. Estas acciones pueden estar orientadas a ejercer la vigilancia en el cumplimiento de la Resolución 1956 de 2008 sobre lugares públicos $100 \%$ libres de humo, y desarrollo y promoción de programas de cesación en Colombia. De la misma manera, se espera poder hacer esta encuesta con una periodicidad de cada cinco años como estrategia de vigilancia y, de ser posible, incluir otras ciudades del país.

\section{Agradecimientos}

Este estudio fue posible gracias a la colaboración de la Organización Mundial de la Salud a través de la Organización Panamericana de la Salud y los Centers for Disease Control and Prevention, quienes diseñaron y coordinan actualmente la Encuesta Mundial de Tabaquismo en Jóvenes como parte del Sistema Global de Vigilancia de Tabaco. Las autoras agradecen la colaboración a los rectores, a los estudiantes de los colegios participantes en la encuesta y a los coordinadores de campo en las ciudades de Bogotá, Bucaramanga, Cali, Manizales y Valledupar.

\section{Conflicto de intereses}

No existe ninguno. 


\section{Financiación}

Este estudio fue realizado con presupuesto de inversión de la nación y recursos técnicos provenientes de la Organización Panamericana de la Salud y los Centers for Disease Control and Prevention.

\section{Referencias}

1. Organización Mundial de la Salud. Informe OMS sobre la epidemia mundial de tabaquismo, 2008. plan de medidas MPOWER. Ginebra: Organización Mundial de la Salud; 2008.

2. Mathers C, Loncar D. Projections of global mortality and burden of disease from 2002 to 2030. PLoS Med. 2006;3:e442.

3. Ministerio de la Protección Social, Instituto Nacional de Cancerología. Hechos y acciones. Boletín. Bogotá: Instituto Nacional de Cancerología; 2004.

4. Instituto de Seguros Sociales, Profamilia. Encuesta sobre conocimientos, actitudes y prácticas. Consumo de tabaco y alcohol. ENFREC I. Tomo VI. Bogotá: Profamilia; 1994.

5. Ministerio de Salud. II Estudio Nacional de Factores de Riesgo de Enfermedades Crónicas - Series Documentos Técnicos. ENFREC II. Bogotá: Ministerio de Salud; 1999.

6. Rodríguez J, Ruiz F, Peñaloza E, Eslava J, Gómez LC, Sánchez H, et al. Encuesta Nacional de Salud, 2007. Bogotá: Javegraf; 2009.

7. Ministerio de la Protección Social, Dirección Nacional de Estupefacientes. Estudio Nacional de Consumo de Sustancias Psicoactivas en Colombia - 2008. Bogotá: Dirección Nacional de Estupefacientes; 2009.

8. Wiesner C, Peñaranda D. Encuesta Mundial de Tabaquismo en Jóvenes. Reporte de Bogotá, Colombia. Rev Colomb Cancerol. 2002;6:5-14.

9. World Health Organization. WHO Framework Convention on Tobacco Control. Geneva: World Health Organization; 2003. Fecha de consulta: 28 de abril de 2009. Disponible en: http://www.who.int/tobacco/framework/WHO_FCTC_ english.pdf.

10. Congreso de la República de Colombia. Ley 1109 de 27 de diciembre de 2006 por medio de la cual se aprueba el "Convenio Marco de la OMS para el control del tabaco", hecho en Ginebra, el veintiuno (21) de mayo de dos mil tres (2003). Diario oficial No. 46.494. 27 de diciembre de 2006. Bogotá: Congreso de la República; 2006.

11. Ministerio de la Protección Social. Resolución 1956 de 30 de mayo de 2008 por medio de la cual se adoptan medidas en relación con el consumo de cigarrillo o tabaco. Diario oficial No. 47.009. 3 de junio de 2008. Bogotá: Ministerio de la Protección Social; 2008.

12. Congreso de Colombia. Ley 1335 de 21 de julio de 2009 por medio de la cual se adoptan "disposiciones por medio de las cuales se previenen daños a la salud de los menores de edad, la población no fumadora y se estipulan políticas públicas para la prevención del consumo de tabaco y el abandono de la dependencia del tabaco del fumador y sus derivados en la población colombiana". Fecha de consulta: 24 de julio de 2009. Disponible en: http://web.presidencia. gov.co/leyes/2009/julio/ley133521072009.pdf.

13. Warren CW, Asma S, Lee J, Lea V, Mackay J. Global Tobacco Surveillance System: The GTSS Atlas. Atlanta: CDC Foundation; 2009.

14. Global Tobacco Surveillance System Collaborating Group. The global tobacco surveillance system (GTSS): Purpose, Production, and Potential. J Sch Health. 2005;75:15-24.

15. Shah BV, Barnwell BG, Bieler GS. Software for the Statistical Analysis of Correlated Data (SUDAAN): User's Manual release 7.5, 1997 (software documentation). Research Triangle Park, NC: Research Triangle Institute; 1997.

16. Warren CW, Jones NR, Peruga A, Chauvin J, Baptiste JP, Costa de Silva V, et al. Global Youth Tobacco Surveillance, 2000-2007. Morbidity and Mortality Weekly Report. Atlanta: CDC, Surveillance Summaries; 2008. p. 57.

17. Ott $\mathbf{C H}$, Cashing SE, Altekruse M. Development and validation of the College Tobacco Survey. J Am Coll Health. 2005;53:231-8.

18. Post A, Gilljam H, Rosendahl I, Meurling L, Bremberg S, Galanti MR. Validity of self reports in a cohort of Swedish adolescent smokers and smokeless tobacco (snus) users. Tob Control. 2005;14:114-7.

19. Dolcini MM, Adler NE, Lee P, Bauman KE. An assessment of the validity of adolescents self-reported smoking using three biological indicators. Nicotine Tob Res. 2003;5:47383.

20. DiFranza J, Wellman R, Sargent J, Weitzman M, Hipple B, Winickoff J. Tobacco promotion and the initiation of tobacco use: Assessing the evidence for causality. Pediatrics. 2006;117:e1237-48.

21. Bravo A, Mantilla L, Osorio M, Martínez V, Alba L, Cendales R, et al. Evaluación de una intervención educativa para prevenir el tabaquismo en escolares colombianos. Rev Colomb Cancerol. 2007;11:228-40.

22. Alba L. Factores de riesgo para iniciar el consumo de tabaco. Rev Colomb Cancerol. 2007;11:250-7.

23. Hoffman BR, Monge PR, Chou CP, Valente TW. Perceived peer influence and peer selection on adolescent smoking. Addict Behav. 2007;32:1546-54.

24. World Health Organization. Gender and tobacco control: a policy brief. Geneva: World Health Organization; 2007. Fecha de consulta: 3 de febrero de 2010. Disponible en: http://www.idrc.ca/uploads/user-S/12097440371policy_ brief.pdf.

25. Campaign for Tobacco-Free Kids. Tobacco industry targeting of women and girls. CTFK Factsheet, 2007. Fecha de consulta: 3 de febrero de 2010. Disponible en: http:// www.tobaccofreekids.org/research/factsheets/pdf/0138.pdf.

26. Congreso de la República de Colombia. Ley 1098 de 2006 "por la cual se expide el Código de la Infancia y la Adolescencia. Fecha de consulta: 28 de abril de 2009. Disponible en: http://www.presidencia.gov.co/prensa_new/ leyes/2006/noviembre/ley1098081106.pdf. 
27. Fichtenberg CM, Glantz SA. Effect of smoke-free workplaces on smoking behaviour: systematic review. BMJ. 2002;27;325:174-5.

28. World Health Organization. Protection from exposure to second-hand tobacco smoke. Policy recommendations. Geneva: World Health Organization; 2007.

29. Ministerio de la Protección Social. Decreto 3039 de 2007 por el cual se adopta el Plan Nacional de Salud Pública 2007-2010. Diario Oficial No. 46.716. 10 de agosto de 2007. Bogotá: Ministerio de la Protección Social; 2007.

30. International Union Against Cancer. Exposure to secondhand smoke. Protecting our children from second-hand smoke. Geneva: International Union Against Cancer; 2008. p. 11-8.
31. Erguder T, Cakir B, Aslan D, Warren CW, Jones NR, Asma S. Evaluation of the use of Global Youth Tobacco Survey (GYTS) data for developing evidence-based tobacco control policies in Turkey. BMC Public Health. 2008;8(Suppl.1):S4.

32. Gillander Gadin K, Hammarström A. Can school-related factors predict future health behavior among young adolescents? Public Health. 2002;116: 22-9.

33. Thomas RE, Perera R. School-based programmes for preventing smoking. Cochrane Database Syst Rev. 2006;3:CD001293.

34. Rangul V, Lingaas T, Kurtze N, Cuypers K, Midthjell K. Reliability and validity of two frequently used selfadministered physical activity questionnaires in adolescents. BMC Med Res Methodol. 2008;8:47. 